\title{
Gastric Diverticulum of the Prepyloric Region: A Rare Presentation of Gastric Diverticulum
}

\author{
T. Tsitsias J.G. Finch \\ Department of General Surgery, Northampton General Hospital, Northampton, \\ UK
}

\section{Key Words}

Gastric diverticulum · Prepyloric region · Proton pump inhibitors

\begin{abstract}
Gastric diverticula are the least common diverticula of the gastrointestinal tract. The two main categories identified are congenital and acquired ones. Diverticula of the antrum, prepyloric or pyloric, are extremely rare and usually asymptomatic. We report a diverticulum of the prepyloric region in a patient with a background of peptic ulcer disease and antiplatelet treatment who became symptomatic and was treated successfully with proton pump inhibitors.
\end{abstract}

\section{Introduction}

Gastric diverticula are the least common diverticula of the gastrointestinal tract and have been observed in $0.03-0.1 \%$ of upper gastrointestinal contrast studies, $0.03-0.3 \%$ of autopsies, and $0.01-0.11 \%$ of oesophagogastroduodenal endoscopies [1]. They usually occur in middle-aged people (between 20 and 60 years of age), with equal distribution among men and women [2]. Gastric diverticula are often single and may vary in shape and size as they can be as large as half of the stomach, although most are 1-6 cm in size. However, multiple and larger diverticula have also been noted [3]. The rapidly increasing use of upper gastrointestinal barium studies has enabled clinicians to identify them promptly and to differentiate them from other pathologies. The majority is found in the posterior wall of the cardia region and along the lesser curvature. However the greater curvature and prepyloric region can be affected as well. Two types of gastric diverticula have been identified: congenital and acquired. 
A large percentage of patients with gastric diverticula will remain asymptomatic throughout life. This is probably explained by their posterior location of the diverticulum and its broad base. Also congenital gastric diverticula being full-thickness true diverticula tend to cause less symptoms and complications than acquired pseudodiverticula. Symptomatic diverticula are rare, and the most common symptoms that patients seek treatment for are upper abdominal pain, nausea, weight loss and emesis. Occasionally, the presentation of a gastric diverticulum can be dramatic related to massive haemorrhage or perforation requiring immediate surgical treatment.

We report the interesting case of a 68-year-old male patient who presented with long-standing symptoms of dyspepsia and upper gastrointestinal tract bleeding that were associated with a gastric diverticulum of the prepyloric region.

\section{Case Report}

A 68-year-old male patient was referred by his general practitioner to the Gastroenterology Outpatient Department, Northampton General Hospital due to on and off symptoms of dyspepsia that started only months after the initiation of aspirin and dipyridamole for a recent lacunar stroke. He experienced occasional heartburn and vomiting for the last year and he also had a few episodes of haematemesis while trying to relieve the reflux symptoms. Shortly after this he had two episodes of melaena. Blood investigations were performed and his urea was raised, but he maintained a satisfactory haemoglobin. His general practitioner initiated an empirical treatment for dyspepsia consisting of the combination of a proton pump inhibitor (PPI) (omeprazole $40 \mathrm{mg}$ ) and an $\mathrm{H} 2$ receptor antagonist (ranitidine $150 \mathrm{mg}$ b.d.) that settled the symptoms for a short period of time but eventually had to be abandoned due to severe diarrhoea. Aspirin was discontinued by the general practitioner and the patient was free of dyspepsia symptoms. He demonstrated a normal appetite and denied any dysphagia.

When seen at the outpatient department by the gastroenterology team, his physical examination demonstrated a soft and nontender abdomen. Bowel sounds were normal and no masses were identified. The remainder of the physical examination was normal. His past medical history included a duodenal ulcer diagnosed more than 40 years earlier that had led to upper gastrointestinal tract bleeding, and he was interestingly found to be one of the pioneers of the first combination treatment for Helicobacter pylori eradication. He also had hypertension, hypercholesterolemia and was a smoker of 10 cigarettes daily. 10 months prior to his referral he had suffered a left hemisphere lacunar stroke with persistent paraesthesia that was treated with double antiplatelet regime (aspirin + dipyridamole). He had no family history of peptic ulceration or upper gastrointestinal bleeding.

An oesophagogastroduodenoscopy (fig. 1) was performed in an attempt to identify the cause of his symptoms and a middle-sized diverticulum was found in the prepyloric region only a few centimetres from the pyloric opening without causing outflow obstruction. The rest of the examination revealed multiple noncircumferential erosions of the oesophagus, without confluence affecting more than one longitudinal fold and suggestive of reflux oesophagitis. The appearance of the duodenum was normal.

Multiple oesophageal biopsies were taken that showed fragments of stratified squamous epithelium with an inflammatory infiltrate including eosinophils and congested capillaries suggestive of reflux oesophagitis, but there was no evidence of dysplasia or malignancy. In view of his past medical history and the nature of his symptoms, a decision was taken to treat the patient conservatively. An alternative PPI agent was commenced (lansoprazole $30 \mathrm{mg}$ o.d.), but aspirin was not restarted since it was considered that dipyridamole would cover him from any further cerebrovascular events with a reduced risk of gastrointestinal bleeding.

He was not given a follow-up appointment for review at the outpatient clinic, but 6 months after the initiation of the PPI treatment he remains asymptomatic and his general practitioner confirms his steady progress. 


\section{Discussion}

Two types of gastric diverticula have been identified, congenital and acquired. Congenital diverticula are more common, comprising $70-75 \%$ of all gastric diverticula [4]. They tend to occur high on the posterior wall of the stomach in the cardia region, approximately $2 \mathrm{~cm}$ below the oesophagogastric junction and $3 \mathrm{~cm}$ from the lesser curvature of the stomach. In most of the cases they are solitary diverticula consisting of all layers of the stomach. Rarely, in paediatric patients pancreatic tissue is found inside the diverticula, suggesting their congenital nature. The pathophysiology of the occurrence of these juxtacardiac diverticula has shown an underlying weakness in the gastric wall resulting from the division of the longitudinal smooth muscle fibres in the cardia region of the stomach [5]. The presence though of gastric diverticula in the antrum, prepyloric or pyloric region is extremely rare.

Acquired (false) gastric diverticula are pseudodiverticula since they are lacking muscular layers and are associated with other conditions such as gastric malignancy, peptic ulcer disease, pancreatitis or prior surgery. They are most common in adults and usually found near the gastric antrum [6]. False diverticula can be further subdivided into the following groups: (a) pulsation type, which may be secondary to conditions associated with increased intraluminal pressure (difficult labour, pyloric obstruction, severe vomiting, coughing, constipation, foreign bodies, etc.) or from the eroding effect of gastric ulcers or carcinoma; (b) traction type, due to perigastric adhesions resulting from inflammatory lesions of the spleen, gallbladder, pancreas, liver, peritoneum [7] or recent surgery of the stomach, i.e. Roux-en-Y gastric bypass.

The definite diagnosis of gastric diverticula is challenging and in the majority of cases is an incidental finding during investigation of their common symptoms. Orally administered barium studies have been used throughout the last decades and helped clinicians to report the first cases in the literature [8]. Their characteristic appearance will reveal a mucosa-lined contrast-filled outpouching with air fluid level in the upright position. On CT scans they are depicted as an abnormal rounded soft tissue shadow in the left paravertebral region. Confusion with adrenal masses, splenic abnormalities or pancreatic tail lesions has been reported [9]. Contrast should be given orally to facilitate diagnosing of gastric diverticula during CT scanning. The rapidly increasing use of upper gastrointestinal endoscopy has enabled us to directly visualize these deformities and also to rule out associated pathology. The use of endoscopic investigations has expanded to intraoperative localization of the diverticulum with placement of the scope or surgical clamp inside the deformity.

Asymptomatic diverticula need no specific treatment. In the majority of asymptomatic cases conservative management can be the first approach. Introduction of PPIs for the treatment of dyspepsia and acid reflux has contributed to the nonsurgical management of these upper gastrointestinal deformities. In cases where only mild symptoms are evident and there are no signs of serious haemorrhage, impending perforation or associated gastric ulcer, PPIs and soft diet will often relieve the symptoms $[2,10]$.

In about $10 \%$ of patients with a true diverticulum surgical treatment is required and should be reserved for gastric diverticula associated with severe complication such as haemorrhage, perforation, chronic inflammation, ulceration and also symptomatic ones 
that demonstrated poor response to the initial medical treatment. Diverticula exceeding $4 \mathrm{~cm}$ are more prone to produce complications and tend to respond less favourably to medication. The optimal surgery is resection of the diverticulum with primary repair [11]. Before the introduction of laparoscopic surgery, the surgical approach was through a median laparotomy or subcostal incision. Even with the open technique, localization of the deformity can be challenging, and reports have described excision of the wrong part of the stomach [12]. An anterior gastrotomy to directly visualize the neck of the diverticulum is required to avoid such pitfalls.

Laparoscopic resection has been shown to be a feasible and safe technique for the treatment of symptomatic gastric diverticula with an excellent outcome [11,13]. Access to the posterior fundus is relatively easy by dividing the gastrocolic ligament (similarly to the approach used during fundoplication). The use of intraoperative gastroscopy or insufflation of the stomach with $0.9 \%$ saline solution via a nasal tube while the antrum is closed has been shown to help localizing gastric diverticula not found with the classic approach [14].

\section{Conclusion}

Gastric diverticula of the prepyloric region are extremely rare and can be associated with peptic ulcer disease. Symptomatic diverticula can be treated medically with initiation of PPIs as long as there is no evidence of severe complications (haemorrhage, perforation). Surgical treatment - open or laparoscopic - should be reserved for such cases. 


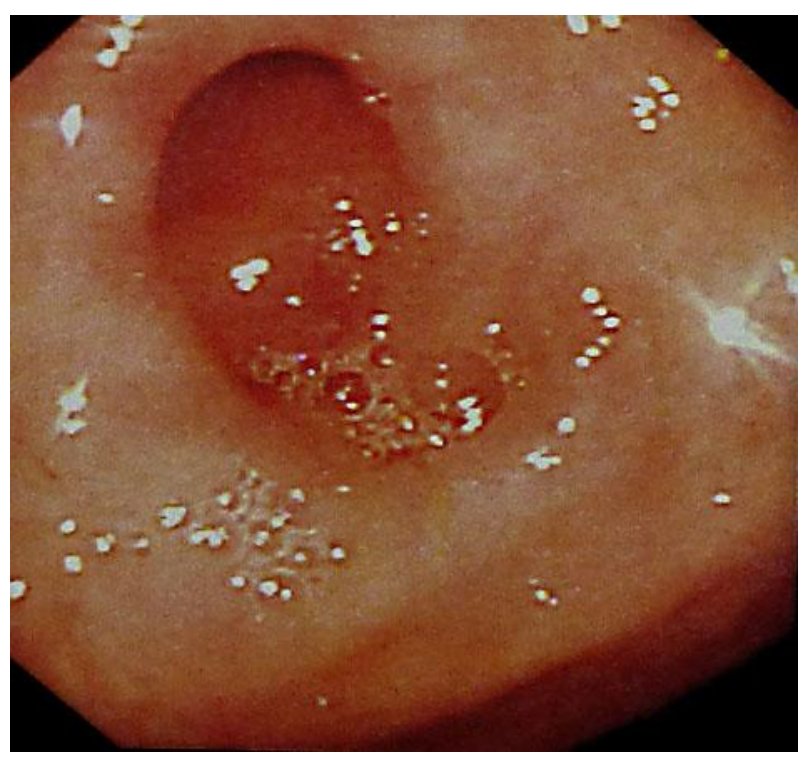

Fig. 1. Oesophagogastroduodenoscopy image. The opening of a middle-sized diverticulum at the prepyloric region was demonstrated. The pylorus adjacent anteriorly to the diverticulum was not obstructed.

\section{References}

1 Simstein NL: Congenital gastric anomalies. Am Surg 1986;52:264-268.

-2 Palmer ED: Gastric diverticulum. Surg Obstet Gynecol 1951;92:417-428.

3 Harford W, Jeyarajah R: Diverticula of the pharynx, esophagus, stomach, and small intestine; in Feldman M, Friedman L, Brandt L, et al (eds): Sleisenger and Fordtran's Gastrointestinal and Liver Disease, ed 8. Philadelphia, Saunders, 2006, pp 465-477.

4 Lopez ME, Whyte C, Kleinhaus S, Rivas Y, Harris BH: Laparoscopic excision of a gastric diverticulum in a child. J Laparoendosc Adv Surg Tech A 2007;17:246-248.

5 Cheng EH, Pavelock RR: Multiple gastrointestinal tract diverticula. Gastrointest Radiol 1990;15: 282-284.

6 Rodeberg DA, Zaheer S, Moir CR, et al: Gastric diverticulum: a series of four pediatric patients. J Pediatr Gastroenterol Nutr 2002;34:564-567.

7 Schmidt HW, Walters W: Diverticula of Stomach. Surg Gynecol Obstet 1935;60:106.

8 Casberg MA, Martin WP: Gastric diverticula. Am J Surg 1948;76:172-181.

$\checkmark 9$ Chasse E, Buggenhout A, Zalcman M, et al: Gastric diverticulum simulating a left adrenal tumor. Surgery 2003;133:447-448.

10 Gockel I, Thomschke D, Lorenz D: Gastrointestinal: Gastric diverticula. J Gastroenterol Hepatol 2004;19:227-229.

11 Fine A: Laparoscopic resection of a large proximal gastric diverticulum. Gastrointest Endosc 1998;48:93-95.

12 Anaise D, Brand DL, Smith NL, Soroff HS: Pitfalls in diagnosis and treatment of a symptomatic gastric diverticulum. Gastrointest Endosc 1984;30:28-30.

13 Vogt DM, Curet MJ, Zucker KA: Laparoscopic management of gastric diverticula. J Laparoendosc Adv Surg Tech A 1999;9:405-410.

14 Donkervoort SC, Baak LC, Blaauwgeers JL, Gerhards MF: Laparoscopic resection of a symptomatic gastric diverticulum: a minimally invasive solution. JSLS 2006;10:525-527. 